\title{
EXOMARS 2016 MISSION ELECTRICAL POWER SYSTEM
}

\author{
Ezio Ciancetta $^{(1)}$, Giuseppe Cuzzocrea $^{(1)}$, Jean-Jacques Digoin $^{(2)}$, Federico Chiusano $^{(3)}$ \\ (1) Thales Alenia Space, Strada Antica di Collegno 253, 10146 Torino (Italy),Ezio.Ciancetta@thalesaleniaspace.com, \\ Giuseppe.Cuzzocrea@thalesaleniaspace.com \\ (2) Thales Alenia Space, 100 Bd du Midi, 06322 Cannes (France), Jean-Jacques.Digoin@thalesaleniaspace.com \\ ${ }^{(3)}$ European Space Agency, Keplerlaan 1, 2201 AZ Noordwijk (The Netherlands), Federico.Chiusano@esa.int
}

\begin{abstract}
ExoMars is the first step of the European Space Agency's Aurora Exploration Programme. Comprising two missions [1], the first one launched in 2016 and the second one to be launched in 2020, ExoMars is a joint ESA-Roscosmos program that will address the scientific question of whether life ever existed on Mars and demonstrate key technologies for entry, descent, landing, drilling and roving on the Martian surface [2].

The Spacecraft Composite (SCC), consisting of a Trace Gas Orbiter (TGO) and an EDL (Entry Descend and Landing) Demonstrator Module (EDM) named Schiaparelli, has been launched on 14 March 2016 from the Baikonur Cosmodrome by a Proton Launcher.

The two modules will separate on 16 October 2016 after a seventh-months cruise. The TGO will search for evidence of methane and other atmospheric gases that could be signatures of active biological or geological processes on Mars and will provide communications relay for the 2020 mission surface assets. The Schiaparelli module will prove the technologies required to safely land a payload on the surface of Mars, with a package of sensors aimed to support the reconstruction of the flown trajectory and the assessment of the performance of the EDL subsystems.

Following separation, the EDM will undergo a threedays coasting phase to reach the Mars atmospheric Entry Interface Point, complete the Entry Descent and Landing phases in six minutes and perform surface science operations for a duration of up to four Martian sols, with data transmission to TGO and to other ESA and NASA orbiters.
\end{abstract}

This paper outlines the Exomars 2016 Electrical Power System (EPS) design, providing a description of the major design drivers and resulting configuration, with a view to highlight aspects that could be considered for future designs.

\section{SPACECRAFT AND MISSION DESCRIPTION}

Thales Alenia Space Italia lead the ExoMars industrial team and they are in charge of EDM design, including the electrical power supply chain. Thales Alenia Space France are responsible for the TGO.

The picture in Fig. 1 shows the SCC in launch configuration.

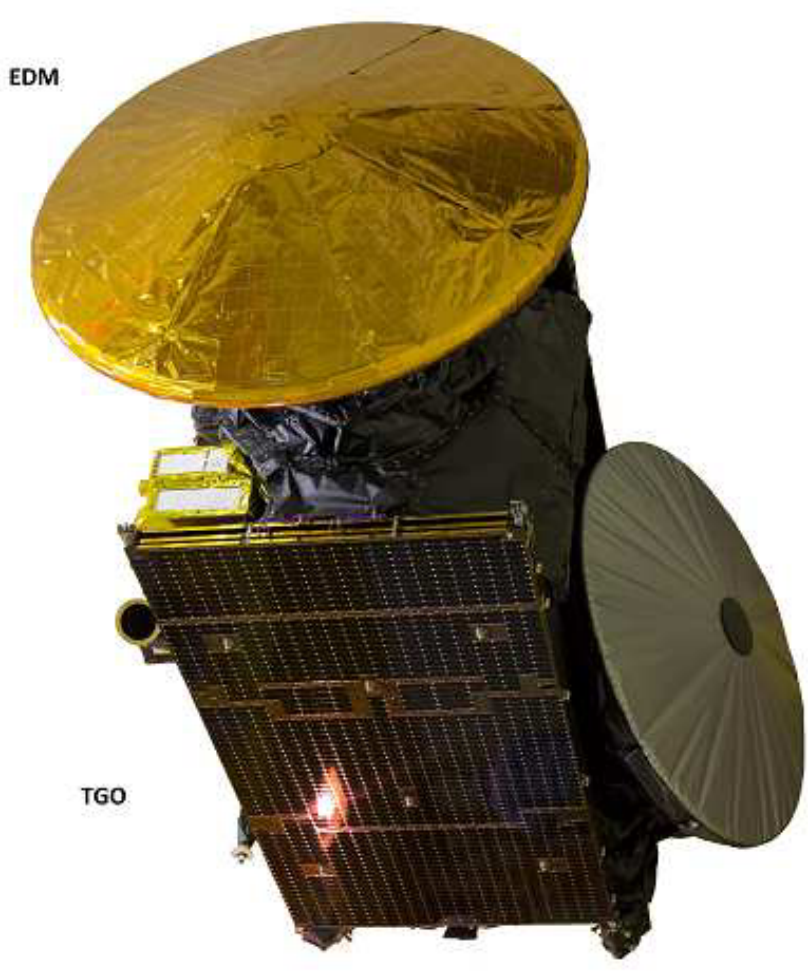

Figure 1. Exomars Spacecraft Composite (SCC) in launch configuration.

The EDM mission will be fully autonomous, with mission phases management performed by On Board Software (OBSW) and the Guidance, Navigation and Control (GNC) in charge of handling mode transitions. GNC functions will be performed in autonomy, without any ground intervention. During the Coasting and EDL phases the EDM power is provided by a set of batteries and GNC functions shall allow the execution of the EDL phase, including the management of two hibernation phases required for energy saving, considering that the entire mission is performed relying on batteries only.

The Exomars 2016 mission phases are shown in Fig. 2. 


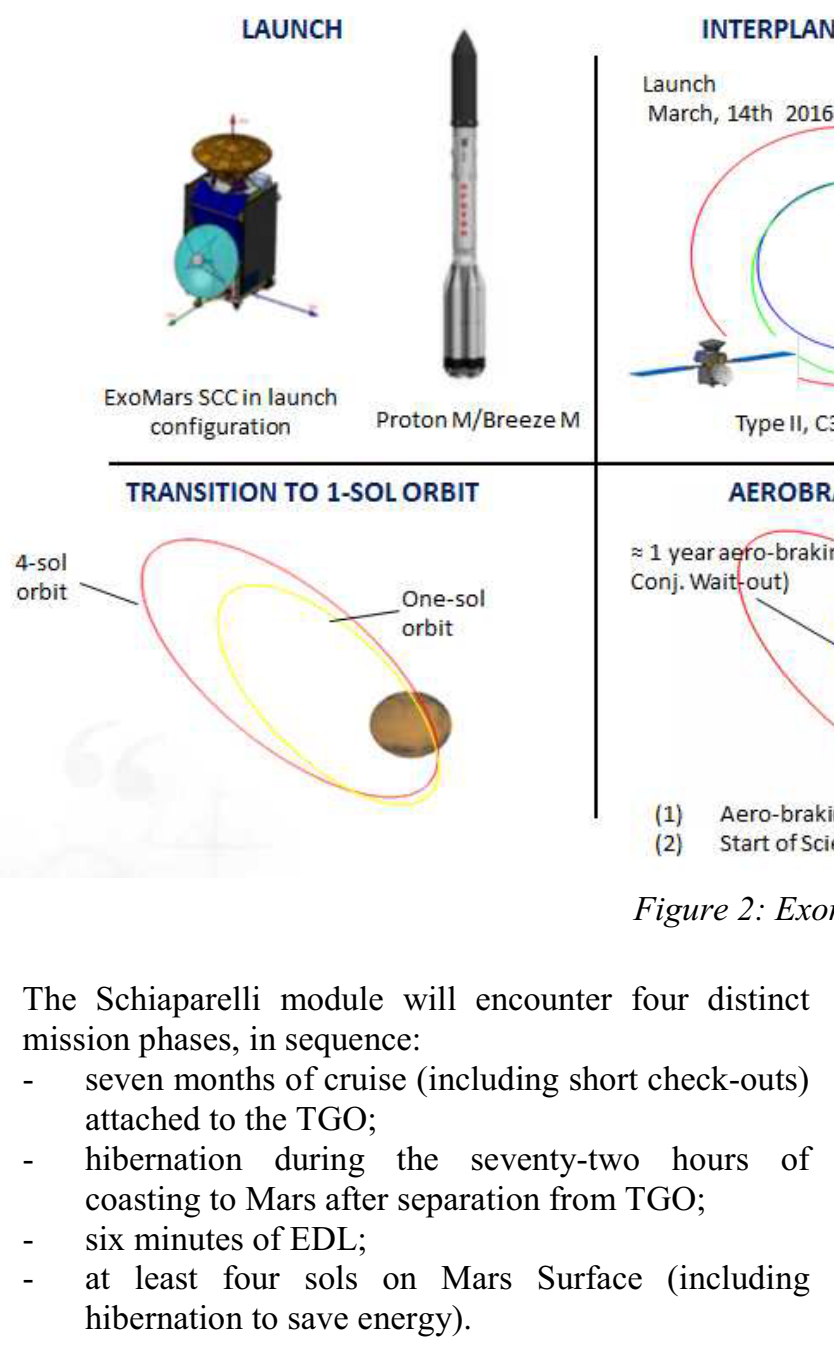

The EDM data will be sent to TGO, to Mars Express orbiter and to the NASA orbiters Mars Reconnaissance Orbiter (MRO), Mars Atmosphere and Volatile Evolution Mission (MAVEN) and Mars Odyssey.

The objectives of the Exomars mission have been modified several times along the history of the program, together with the changes of the international partners. As a consequence, the spacecraft architecture was reconsidered accordingly, with changes affecting also the power supply chain.

\section{EDM ELECTRICAL POWER SUBSYSTEM DESIGN DRIVERS AND ARCHITECTURE}

The Electrical Power System architecture was built around some major design drivers; in particular, the following aspects have been considered for defining the design of the EDM Power system.

- Reception of power from the TGO. During the cruise phase, the power system has to be capable of receiving power from the Orbiter.

mission phases, in sequence:

attached to the TGO;
hibernation during the seventy-two hours of

- six minutes of EDL;

at least four sols on Mars Surface (including
APPROACH, EDM RELEASE \& MOI

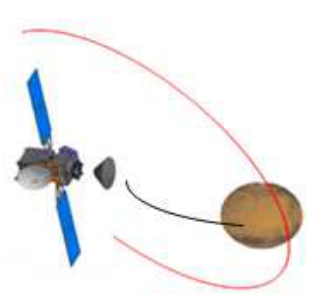

SCIENCE \& DATA RELAY PHASE

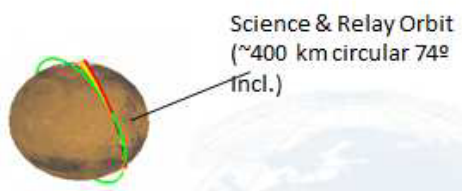

(1) Data relay for 2020 Rovers and Surface Platform

(2) Data relay capability for future Mars surface assets throughout 2022 (n) allowing the battery recharge, only primary power sources can be used and therefore the maximization of power density (maximum ratio between power and mass) was necessary. The first selection was using primary cells for the batteries, but the energy stored inside any single battery cell was too high and risky for the mission, in particular for the mechanical loads. In fact, during a vibration test a primary cell exploded as a consequence of internal short circuit.

- Optimization of the power consumption. Having a reduced power source, a strong optimization in the power consumption was required. All units were specified to be low power demanding. A hibernation mode was designed for the periods in which the capsule does not need a complete functionality, e.g. in the three days approaching Mars and during the surface operations in all the time frames when the communications with orbiters are not possible. In this mode, only timers are active to wake up the EDM when required.

- Thermal insulation to minimize the power 
consumption for heating the vital units. All the vital units needed on the surface are located in a specific Warm Compartment highly isolated from the external environment. The Battery temperatures have to be kept sufficiently warm to optimize their energy production.

The EDM EPS is designed to be powered by the TGO unregulated power bus or by its internal batteries. The EPS power conditioning and distribution functionalities are implemented into two different units: Central Terminal and Power Unit (CTPU) and Remote Terminal and Power Unit (RTPU). The CTPU implements the part of the EDM EPS that is requested to be operative also on Mars surface and for this reason it is located in the EDM Warm Compartment. The RTPU indeed is located on the crushable side of the lander and it is no longer used after touch-down on Mars surface.

The EPS block diagram is shown in Fig. 3.

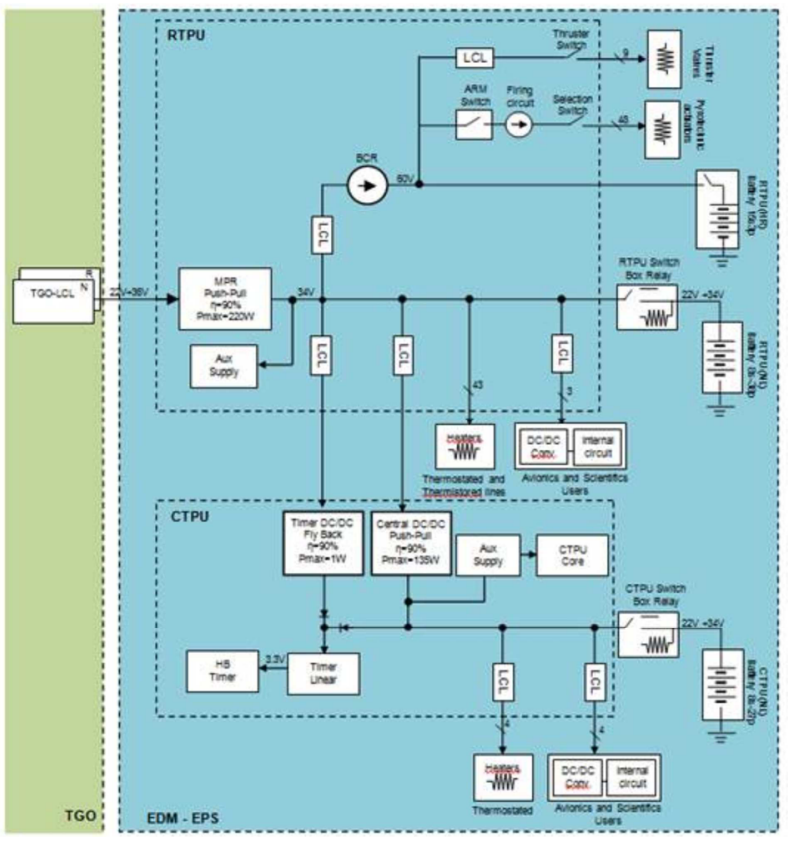

Figure 3. EDM EPS Block Diagram.

The RTPU Main Power Regulator (MPR) is fed by the TGO unregulated power bus and regulates it to a stable voltage of $34 \mathrm{~V} \pm 0.5 \%$, with a power capability of $220 \mathrm{~W}$ and an efficiency greater than $90 \%$.

The CTPU Central DC/DC converter regulates the power coming from RTPU Main Bus to $34 \mathrm{~V} \pm 2 \%$, with an efficiency greater than $90 \%$ at $135 \mathrm{~W}$. These regulations are needed to guarantee a bus voltage slightly higher than End of Charge (EoC) battery voltage, i.e. $33.6 \mathrm{~V}$.

The CTPU and RTPU regulated bus are distributed via Latching Current Limiters (LCL) to the EDM equipment.

The EPS regulated bus stability has been designed in accordance with the Middlebrook criteria [3] and it has been successfully tested on the EDM Avionic Test Bench (ATB) and Proto-Flight Model (PFM).

An exploded view of the EDM is reported in Fig. 4. The location of EPS equipment is shown in Fig. 5.

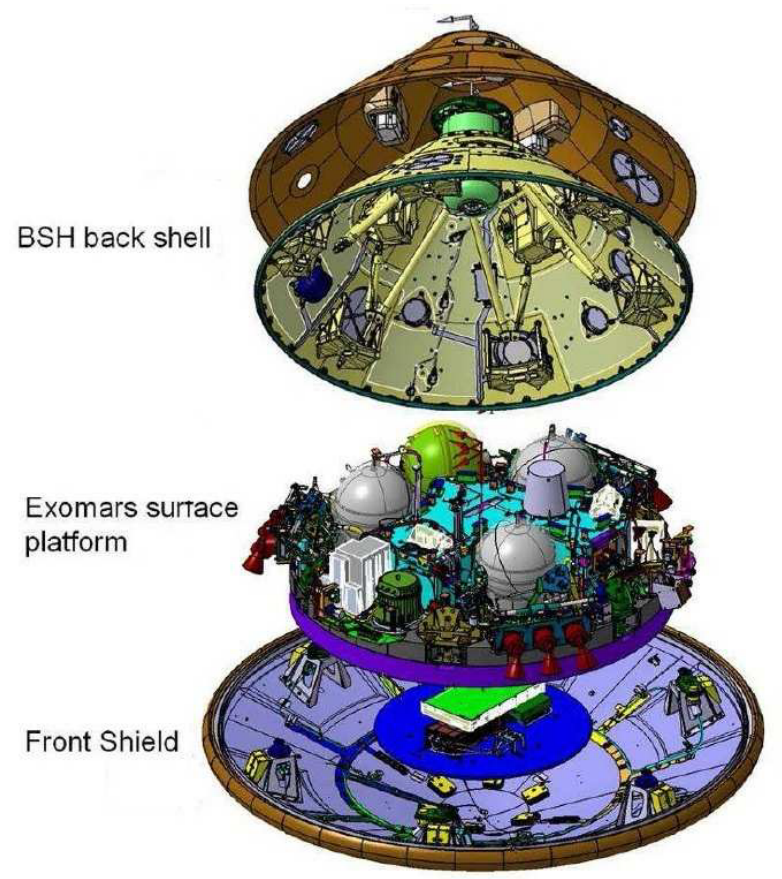

Figure 4. EDM exploded view.

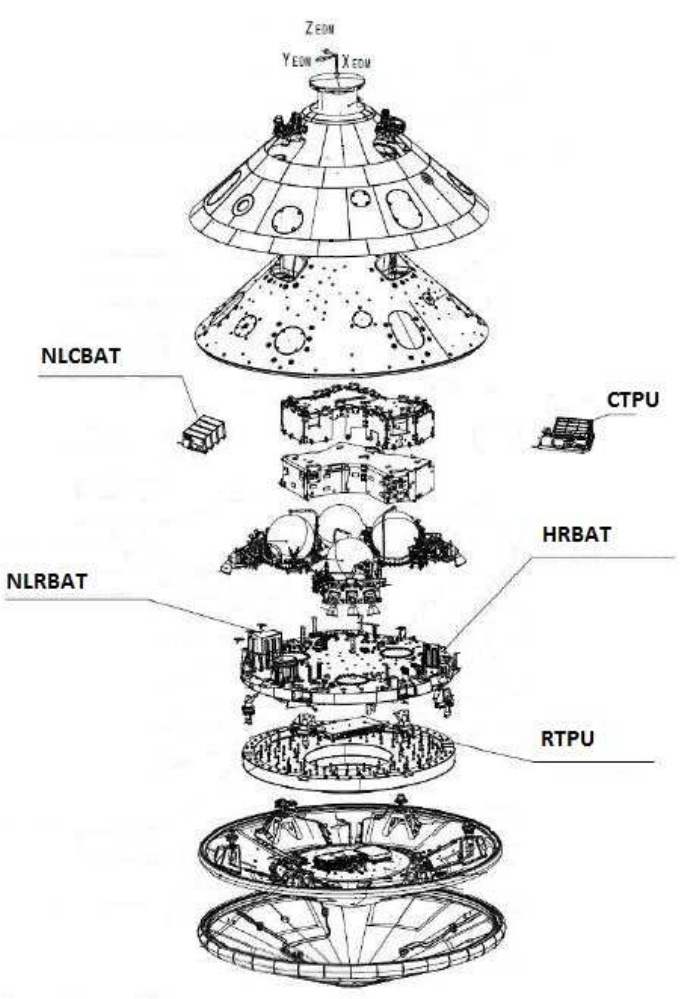

Figure 5. EDM EPS equipment configuration. 
During the cruise the RTPU is nominally switched ON (EDM service mode), while the CTPU is operative only during check out phases and during the EDM Batteries recharge phase. When the EDM is in service mode the TGO is committed to manage the EDM Thermal Control System (TCS), executing in its Satellite Management Unit (SMU) the EDM TCS algorithm. This algorithm, especially in coldest condition, is aimed to tailor the TCS consumption as complement of avionic units power demand, keeping the total power below the RTPU MPR capability.

The EDM batteries recharging phase is scheduled at the end of the cruise, in order to compensate the slight selfdischarge of the lithium cells.

Three different battery modules, based on lithium cells, have been designed to supply the EDM when it switches to internal power mode:

- RTPU NL Battery (NLRBAT - 87Ah), used in Coasting and EDL phase;

- CTPU NL Battery (NLCBAT - 62Ah), used on Mars surface;

- $\quad$ RTPU High Rate (HR) Battery (HRBAT - 3.3Ah), used to supply during the EDL the $60 \mathrm{~V}$ unregulated power bus of the RTPU, used for pyro firing and RCS actuation with a peak current capability of $45 \mathrm{~A}$ as required during pyro actuation.

The HRBAT is connected to the $60 \mathrm{~V}$ unregulated bus via latching relays embedded into the battery. The charging is managed via a dedicated Battery charge Regulator (BCR) with three selectable levels of EoC voltage (up to $61.5 \mathrm{~V}$ ) and charge current rate (up to $0.3 \mathrm{~A}$ ). The converter has a global efficiency greater than $80 \%$ and it implements the taper mode functionality.

The NLRBAT connection to Main Bus (MB) is managed via relay boxes. The charge functionalities are implemented by power resistors, bypassed when the EDM is in internal power mode. The simplicity of the NL battery charge system is paid in terms of charge time. Fig. 6 shows the test results of NLRBAT charged via $4.99 \Omega$ resistor using a bus voltage of $34.5 \mathrm{~V}$. The charge resistor is bypassed at $34 \mathrm{~V}$ and the battery is directly connected to the MB. This design solution has been chosen because the need of recharging this battery was specified when the design was already frozen.

At the separation from the TGO the EDM is switched in internal power, then the EDM goes in coasting hibernation mode. In this phase in order to save energy the RTPU and CTPU Main and Aux converter are switched OFF and the RTPU main bus is handled by NLRBAT. This unregulated bus is distributed via LCLs to heater lines and CTPU. The RTPU distribution losses in this phase are lower than $1 \mathrm{~W}$. The exit from hibernation is scheduled by CTPU.

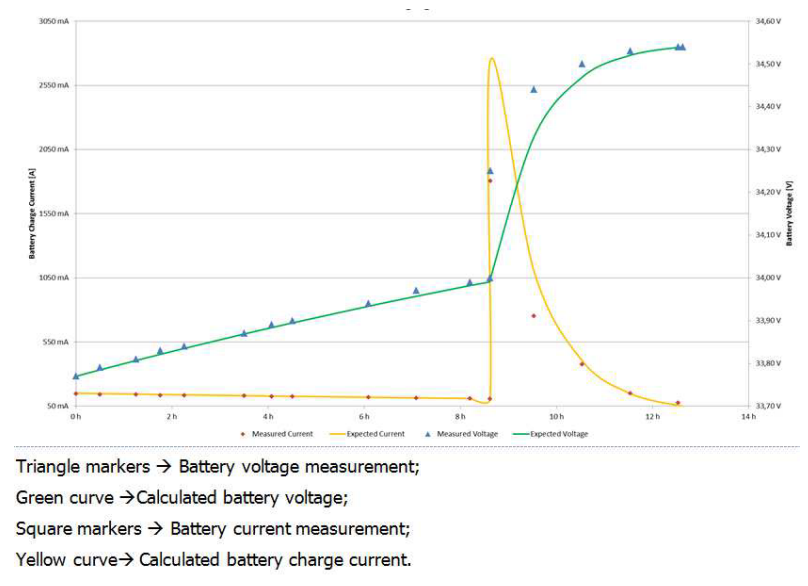

Figure 6. NLRBAT Charge model vs. measurements.

The CTPU Timer logic is powered by dedicated low power converter. The Timer DC/DC converter takes power from the RTPU unregulated bus and regulates it to $34.5 \mathrm{~V} \pm 5 \%$ with a power capability of $1 \mathrm{~W}$ and an efficiency greater than $90 \%$. During coasting hibernation, the overall CTPU power consumption is less than $1 \mathrm{~W}$ and the most part of the energy consumed by EDM is spent by the thermal control.

The coasting discharge power profile of NLRBAT has been simulated using EDM correlated thermal model with an overall system margin of 5\%, as shown in Fig. 7. The NLRBAT is predicted to complete the mission with a final State of Charge of $16 \%$.

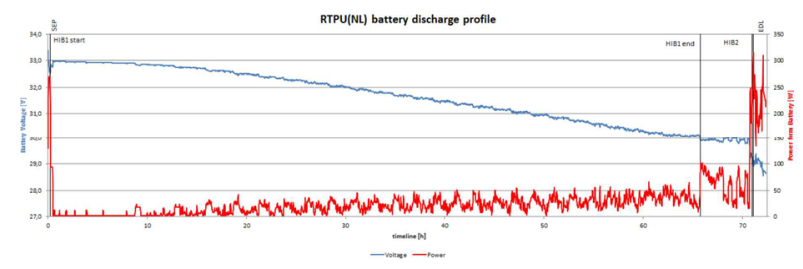

Figure 7. NLRBAT Simulated Discharge Profile.

During Entry, Descent and Landing the HRBAT is in charge to provide high power peaks, with duration of 25 to $50 \mathrm{~ms}$, demanded by pyrotechnic actuators used in propellant circuit valve (up to $360 \mathrm{~W}$ ), Parachute Deployment Device (up to $720 \mathrm{~W}$ ), front shield and back shell separation mechanism (up to $1800 \mathrm{~W}$ ). These performances are met by the HRBAT even with a string in failure.

The main part of the HRBAT energy is spent to supply the Reaction Control System (RCS) flow control valve during breaking manoeuvre at the end of the parachute phase (up to $800 \mathrm{~W}$ for $60 \mathrm{~s}$ ).

Fig. 8 shows how the under-voltage condition $(48 \mathrm{~V})$ is never triggered on the RTPU $60 \mathrm{~V}$ unregulated bus. The TCS is designed to maintain the battery temperature always above $10^{\circ} \mathrm{C}$ in order to avoid battery Internal Resistance (IR) degradation. 


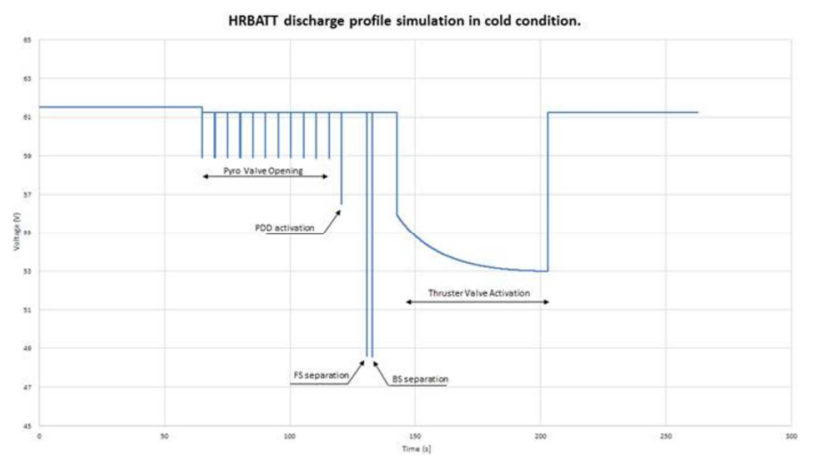

Figure 8. HRBAT Discharge Profile.

On Mars surface the lander is fed by NLCBAT which is accommodated in the Warm Compartment together with Transceiver (TVCR), CTPU and scientific equipment. The relay box connecting the NLCBAT to the main bus is mounted outside the Warm Compartment, being able to withstand Mars coldest temperature.

The DREAM (Dust characterisation, Risk assessment and Environment Analyzer on Martian Surface) Instrument units are powered autonomously by a dedicated battery.

During Mars surface operation the lander is always in Hibernation except during orbiter passes (30 min), where CTPU and the TVCR are fully operative in order to upload the scientific data. The most part of NLCBAT is used by thermostated heater lines, being the CTPU power dissipation lower than $1 \mathrm{~W}$ : this is because only the $3.3 \mathrm{~V}$ linear regulator is working.

The discharge power profile of NLCBAT during Mars surface operations has been simulated using EDM correlated thermal model with an overall system margin of $5 \%$, as shown in Fig. 9. The greater contributor in terms of power request is the TCS, that depends of course from the external environment not totally predictable at priori. Therefore in case of very benign external environmental conditions, where the temperature is warmer than expected, the life of Mars can be increased up to ten sols.

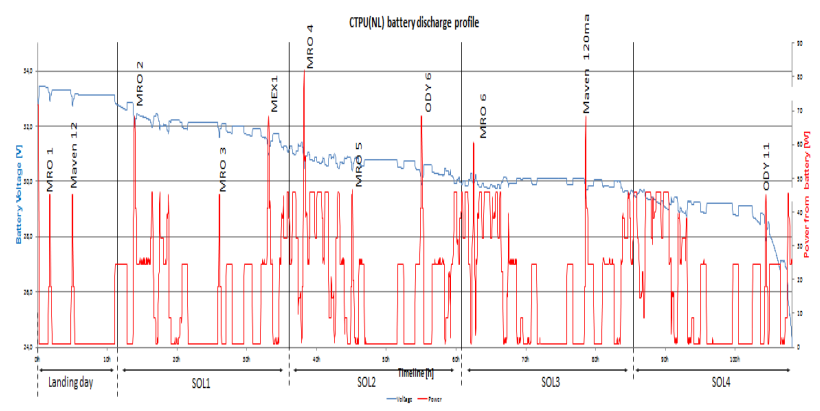

Figure 9.NLCBAT simulated discharge profile.
The NLCBAT is predicted to supply the lander operation through the eleven orbiter passes scheduled in the $98 \mathrm{~h}$ (landing day +4 sols) following the touch down on Mars surface with the standard thermal environment.

\section{TGO ELECTRICAL POWER SUBSYSTEM DESIGN DRIVERS AND ARCHITECTURE}

The TGO EPS is in charge of electrical power generation and energy storage for the Exomars spacecraft composite during launch, cruise and Mars orbiting phases.

EPS design drivers are the variable distance to Sun, the numerous phases associated to different demands in power (with and without telemetry transmission or with and without UHF communication for examples), the occurrence of solar eclipses with varying durations (from few minutes up to 160 minutes).

The Power Subsystem is sized taking into account nominal and contingency situations, against the two most critical scenarios which are the following:

- during the Aerobraking phase, at the end of the sequence where orbit durations are the shortest and the off-sun durations (eclipses + atmospheric passes) the longest;

- $\quad$ in Science phase, taking into account the optimized communication scenarios (with the associated Telemetry Tracking and Command subsystem consumption in Tx mode), as the eclipses may span over $36 \%$ of the orbit.

The Power Subsystem shall also withstand the launch phase. In March 2016 scenario the duration of this phase, from launcher lift-off up to solar array deployment, had a duration around 11 hours. During this phase the TGO was spinned and the stowed solar arrays (see Fig. 1) were alternately Sun pointed (one panel faced to Sun) during a period of 600 seconds. This sequence is called "Flip-Flap" sequence. Even if the Battery is sized to supply the TGO during all this sequence with the predicted thermal environment, the recharging during the Sun pointed periods were appreciated to reduce the Depth of Discharge (DoD).

The TGO EPS provides the following power via an unregulated bus to the whole SCC:

- $\quad$ Power needed by TGO, including

- $190 \mathrm{~W}$ to the Science Payloads

- $55 \mathrm{~W}$ to Science Payloads survival heaters (Payloads Off).

60W to EDM during Cruise,

- $120 \mathrm{~W}$ to EDM during EDM commissioning and health check,

- $240 \mathrm{~W}$ to EDM during thermal boost before separation,

- $120 \mathrm{~W}$ to the Main Separation Assembly (MSA) 


\section{TCS heaters up to EDM separation.}

The overall TGO power loads, not including battery recharge, is variable along the mission phase and configuration and could reach up to $1200 \mathrm{~W}$ in worst case.

During all the mission phases, in order to reduce its recharge duration, the battery will be recharged with the maximum available current. Charge current limit is $60 \mathrm{~A}$, allowing a power recharge up to $2000 \mathrm{~W}(\mathrm{C} / 3)$.

Exomars TGO EPS is composed of the following equipment:

- Li-Ion battery (BTY), composed in baseline of two identical modules, having an overall name-plate capacity of $180 \mathrm{Ah}$;

- Power Conditioning Unit (PCU), including the solar array power conditioning performed by Maximum Power Point Tracking (MPPT) Buck regulators and the battery management;

- Propulsion Control and Distribution Unit (PCDU), including the protection and distribution functions, the pyro commands and the RCS interfaces;

- Solar Array (SA), composed of two deployable wings, each wing equipped of two Ga-As panels, one yoke panel (not cells populated) and one SADM mechanism;

- Harness, providing the interconnections between all the electrical and electronic equipment installed on the TGO, down to the EDM, the Payloads electrical interfaces and umbilical interface.

The TGO EPS architecture is depicted in Fig. 10.

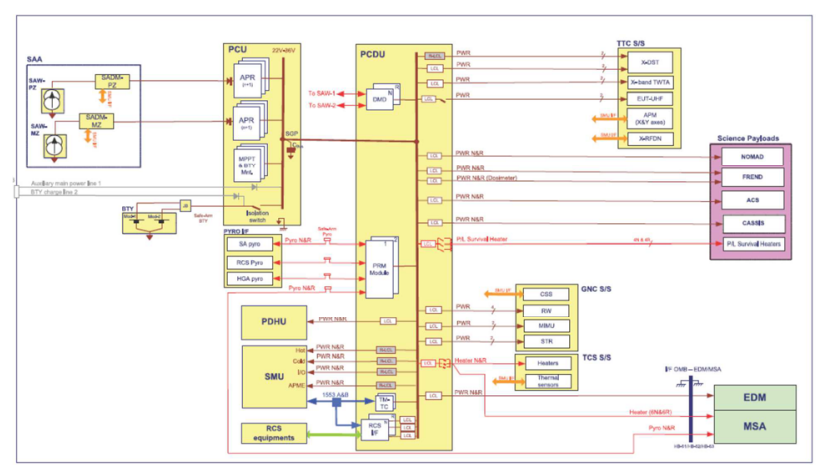

Figure 10. TGO EPS Block Diagram.

The power conditioning functions are totally autonomous and no reconfiguration is expected from the Satellite Management Unit (SMU) in case of single failure. In particular, hot-redundant electronics have been implemented for the power conditioning and battery management functions.
The PCDU distributes an unregulated and protected bus voltage (by means of R-LCL/LCL) to the TGO avionic units, the EDM and the four payloads.

The nominal bus voltage range is $22 \mathrm{~V} \div 36 \mathrm{~V}$ defined as follows (measured at PCU output level):

- Maximum operating voltage: 36V (maximum voltage when the battery is in recharge at its maximum EOCV);

- Minimum operating voltage: 22V (lower limit in a safe mode)

The maximum non-operating voltage is $40 \mathrm{~V}$, maximum voltage to be withstood by user units.

The Solar Array is a major equipment of the orbiter module bus: its sizing is of particular importance and shall consider the complexity of the mission, in particular the aerobraking sequence.

The aerobraking is a manoeuvre performed near Mars (about $120 \mathrm{~km}$ of pericenter height) to reduce the orbital period. It consists of several manoeuvres (or passes, about 800 during the overall sequence) through the upper level of the Mars atmosphere. The heat generated by the friction of the spacecraft against the atmosphere dissipates the kinetic energy of the spacecraft, thus imparting a change in velocity or delta-V.

The Solar Array rear face will be exposed to the dynamic flux during each aerobraking pass (up to $2800 \mathrm{~W} / \mathrm{m}^{2}$ ). The thermal analysis verified that sensitive elements (e.g. cells, substrate, blocking diodes,...) do not exceed their qualified temperature. The analysis results show that the maximum temperatures are reached during the walk-out (end of aerobraking, lasting two weeks).

Fig. 11 shows the deployment of TGO solar array in TAS Cannes facilities.

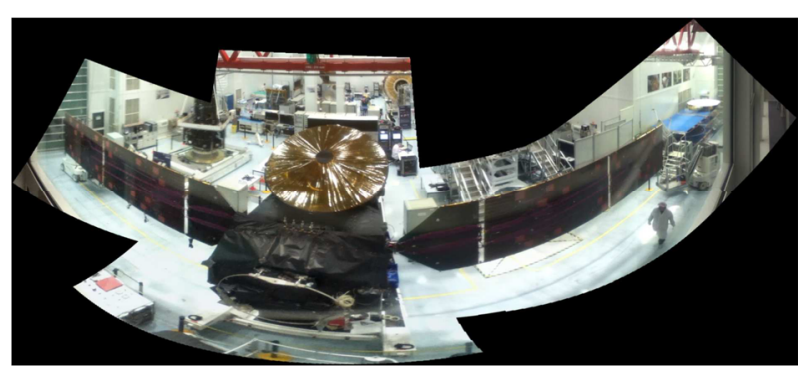

Figure 11. Panoramic photo of the deployed solar array integrated on TGO in Cannes facilities.

The location of TGO power equipment is shown in Fig. 12. 


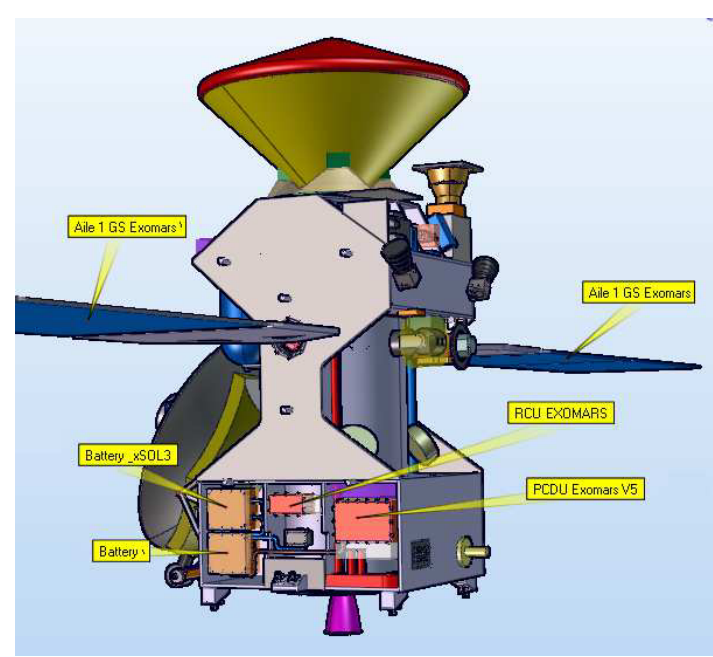

Figure 12. TGO configuration, showing the EPS units location.

Additional data relevant to TGO EPS were reported in [4] and [5].

In order to simulate the different ExoMars phases and verify the correct EPS behaviour, a specific tool has been developed by TAS. Some simulation results are depicted in the figures below.

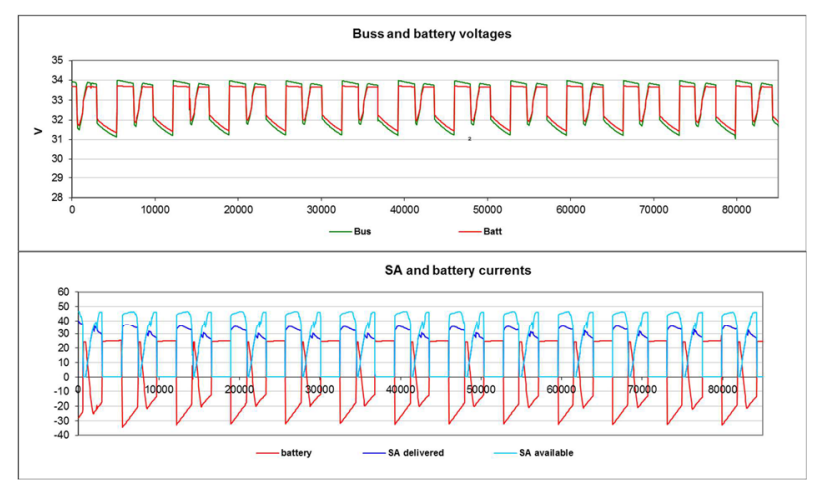

Figure 13. Results of walk-out simulations.

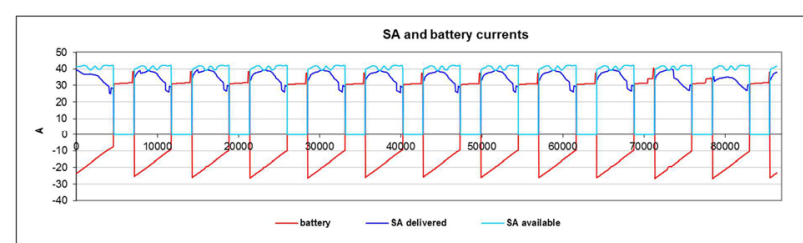

Figure 14. Results of science phase simulations.

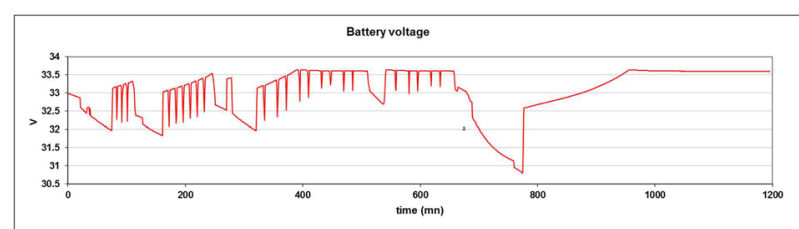

Figure 15. Result of battery voltage simulation during Flip-Flap.

These simulation results have also been checked with flight telemetries and showed a good correlation. An example of battery voltage is reported in the following figure.

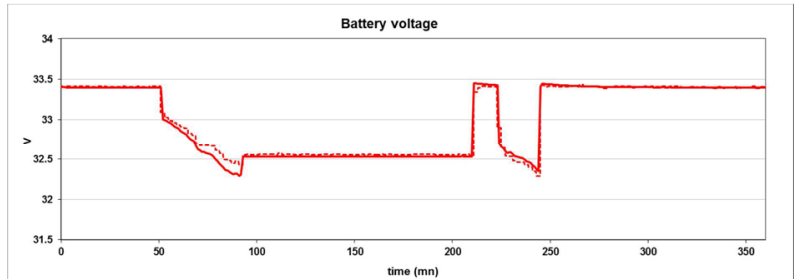

Figure 16. Battery voltage (red bold simulation, red doted TM).

\section{CONCLUSIONS}

This paper presented the Exomars 2016 mission Electrical Power System, describing the major design drivers, the architecture and the results of the preliminary measurements performed in flight.

At the time of the issue of this paper, the Launch and Early Orbit Phase has been completed with a successful In Orbit Commissioning Review. The Deep Space Manoeuvres (DSM 1 and 2) have been successfully performed at the end of July and mid-August and have put the Spacecraft in the correct orbit for Mars. The landing on Mars surface of the Schiaparelli module is foreseen on 19 October 2016 at about 14:47 GMT. At that time the TGO will perform the critical Mass Orbit Insertion (MOI) manoeuvre.

\section{REFERENCES}

1. Cassi, C., Vinai, B., Gianfiglio, G. \& Baglioni, P. (2012). ExoMars: One Project Two Missions. In Proc. $63^{\text {rd }}$ International Astronautical Congress, IAC-12.A3.3A.3.

2. Capuano, M., Portigliotti, S., Fiumanò, A., Blancquaert, T., Bayle, O. \& Lorenzoni, L.. Exomars Mission 2016: EDM Science Opportunities. In Proc. $63^{\text {rd }}$ International Astronautical Congress, IAC-12.A3.3A.4.

3. Middlebrook, R.D. (1976). Input filter considerations in design and application of switching regulators. In Proc. IEEE IAS annual meeting, 1976.

4. Digoin, JJ. \& Boutelet, E. (2011). Exomars Mission 2016, Orbiter Module Power System Architecture. In Proc. $9^{\text {th }}$ European Space Power Conference, ESA SP-690 (CD-ROM), European Space Agency, Noordwijk, The Netherlands.

5. Croci, L., Zanella, P. \& Riva, S. (2014). The Exomars Trace Gas Orbiter Electrical Power Subsystem. In Proc. $10^{\text {th }}$ European Space Power Conference, ESA SP-719 (CD-ROM), European Space Agency, Noordwijk, The Netherlands. 